\title{
APPLICATION OF HM-NETWORKS WITH INPATIENT CLAIMS IN FINDING THE MEMORY CAPACITY IN INFORMATION SYSTEMS
}

\author{
Mikhail Matalytski, Pawel Zajac \\ Institute of Mathematics, Czestochowa University of Technology \\ Czestochowa, Poland \\ m.matalytski@gmail.com,pawel_zajac@vp.pl
}

Received: 6 March 2017; Accepted: 14 August 2017

\begin{abstract}
To solve the problem of determining the memory capacity of the information systems (IS), the use of the stochastic model is proposed, based on the use of HM (Howard-Matalytski) - queueing networks with revenues. This model allows one to take into account time dependencies of the message processing from their capacities, the possibility changes of the messages capacities over time and also the possibility of leaving messages from queues in nodes of IS, without getting into them appropriate processing. The expressions for the mean (expected) values of total message capacities in the IS nodes have been obtained.
\end{abstract}

MSC 2010: $49 K 45,60 K 20$

Keywords: HM-networks, information systems, inpatient claims, capacities of claims

\section{Introduction}

In the IS, the total amount of memory capacity is bounded by some value, which is usually called memory capacity [1]. In the IS, designing the main task is the determination of the mean memory capacity so as to take into account the conditions that limit the proportion of the lost information. One of the methods for solving problems in IS design is the use of HM-queueing networks [2]. Furthermore, under the IS we will mean systems, objects of transformation in which is information, incoming portions as messages [1]. HM-networks can be used to determine the capacity of a buffer storage of systems that are representing processing IS nodes and transferring messages. Note that the considered problem is one of the main ones, for example, in the design of communication centers or hubs in data communication networks. Neglecting time dependence of the message processing from their capacity can lead to errors in determining the buffer capacity memory in the IS and in the calculation of the probability of message loss. 
The model expounded below can also be used in solving the actual problem that has emerged recently in the IS, namely, excessive buffering problems (i.e. the definition of the required memory capacity) [3]. Bufferbloat - a phenomenon that occurs in packet communication networks, when the buffering causes excessive increase in the time of the packets and packet delay variation, and the resulting decrease in throughput capacity of IS.

Neglect time dependence of messages processing of their capacities can lead to serious errors in finding the buffer memory in IS. In the general case solving the problems discussed above can be based on the use of HM-networks with revenues. In such networks, the claim during the transition from one queueing system (QS) to another brings some revenue last (which is equal to the capacity of this claim), and revenue (capacity) of the first QS is reduced by this amount.

It should be noted that the method for finding the stationary state probabilities and the mean characteristics of the queueing network with bounded time of the same type claims, operating under a heavy traffic regime, using the apparatus of multivariate generating functions, has been described in the monograph [4] and to the network with heterogeneous claims - in [5], HM-networks with bounded waiting time of claims have been studied in [6,7].

For the first time application of the HM-networks for estimating the memory capacity in the IS has been described in [8]. In [9] a method of finding the mean total capacity of the same type claims in open systems of HM-network with a bounded their numbers of queues and claims bypassing of queueing systems.

\section{Finding the expected capacities of claims in the case, when is known only the first moments of the revenues from the transitions between the network states}

Let an independent Poisson flow arrive to the network with rate $\lambda$. The intensity of service of claims at time $t$ has rate $\mu_{i}\left(k_{i}(t)\right)$ in the system $S_{i}$ and depends on the count of claims in this system, $i=\overline{1, n}$. The length of stay of claims in the queue of the $i$-th QS is a random variable, distributed exponentially with parameter $\theta_{i}\left(k_{i}(t)\right)$, and does not depend on other factors, for example, the residence time in the queue of other claims.

Consider the dynamics of income changes of a network system $S_{i}$. Denote by the $V_{i}(t)$ its income at moment time $t$. Let the initial moment time revenue of the system equal $V_{i}(0)=v_{i 0}$. The revenue of its QS at moment time $t+\Delta t$ can be represented in the form

$$
V_{i}(t+\Delta t)=V_{i}(t)+\Delta V_{i}(t, \Delta t)
$$


where $\Delta V_{i}(t, \Delta t)$ - revenue changes of the system $S_{i}$ at the time interval $[t, t+\Delta t)$, $i=\overline{1, n}$. To find its value, we write down the value of the conditional probabilities of events that may occur during $\Delta t$ and the revenue changes of its QS, associated with these events:

1) With probability $\lambda p_{0 i} \Delta t+o(\Delta t)$ to the system $S_{i}$ will arrive a claim from the external environment, which will increase its total capacity of a claim by a value $r_{0 i}$, where $r_{0 i}$ - a random variable (RV) with the expectation $E\left\{r_{0 i}\right\}=a_{0 i}$, $i=\overline{1, n}$.

2) With probability $\mu_{i}\left(k_{i}(t)\right) u\left(k_{i}(t)\right) p_{i 0} \Delta t+o(\Delta t)$ a claim after it has been serviced in QS $S_{i}$ is headed for the external environment, wherein total capacity of claims in the system $S_{i}$ reduced by the amount of $R_{i 0}$, where $R_{i 0} \mathrm{RV}$ with $E\left\{R_{i 0}\right\}=b_{i 0}$.

3) With probability $\mu_{j}\left(k_{j}(t)\right) u\left(k_{j}(t)\right) p_{j i} \Delta t+o(\Delta t)$ a claim, after servicing in the QS $S_{j}$, heads for the system $S_{i}$, in such a transition claim capacity in the system $S_{i}$ increases by a value $r_{j i}$, and claims capacity in the system $S_{j}$ reduced by this value, where $r_{j i} \mathrm{RV}$ with $E\left\{r_{j i}\right\}=a_{j i}, i, j=\overline{1, n}, i \neq j$.

4) With probability $\mu_{i}\left(k_{i}(t)\right) u\left(k_{i}(t)\right) p_{i j} \Delta t+o(\Delta t)$ a claim from the system $S_{i}$ heads for the system $S_{j}$, in such a transition a claim capacity of the system $S_{i}$ reduced by the value $R_{i j}$, and claims capacity in the system $S_{j}$ will increase by this value, where $R_{i j}-\mathrm{RV}$ with $E\left\{R_{i j}\right\}=b_{i j}, i, j=\overline{1, n}, i \neq j$. It's obvious that $r_{j i}=R_{j i}$ with probability 1 , i.e.

$$
a_{j i}=b_{j i}, i, j=\overline{1, n} .
$$

5) With probability $\theta_{i}\left(k_{i}(t)\right) u\left(k_{i}(t)\right) q_{i 0} \Delta t+o(\Delta t)$ a claim without waiting for service in the system $S_{i}$, will moves from the queue of this QS to the external environment, and claims capacity in it reduced by the value $H_{i 0}$, where $H_{i 0}$ - RV with $E\left\{H_{i 0}\right\}=\bar{H}_{i 0}, i=\overline{1, n}$.

6) With probability $\theta_{j}\left(k_{j}(t)\right) u\left(k_{j}(t)\right) q_{j i} \Delta t+o(\Delta t)$ a claim without waiting for service in the system $S_{j}$, moves from the queue of this QS to the QS $S_{i}$, in such a transition claims capacity in it will increase by a value $h_{j i}$, and claims capacity in the QS $S_{j}$ reduced by this value, where $h_{j i}$ - RV with $\bar{h}_{j i}, i, j=\overline{1, n}, i \neq j$. 
7) With probability $\theta_{i}\left(k_{i}(t)\right) u\left(k_{i}(t)\right) q_{i j} \Delta t+o(\Delta t)$ a claim without waiting for service in the system $S_{i}$, moves from this QS to the system $S_{j}$ in such a transition a claim capacity of the system will increase by a value $H_{i j}$, and claims capacity in the QS $S_{i}$ reduced by this value, where $H_{i j}$ - RV with $\bar{H}_{i j}$; it's clear that $h_{j i}=H_{j i}$ with probability 1 , i.e.

$$
\bar{h}_{j i}=\bar{H}_{i j}, i, j=\overline{1, n} .
$$

8) With probability

$$
\begin{gathered}
1-\left[\lambda p_{0 i}+\left(\mu_{i}\left(k_{i}(t)\right)+\theta_{i}\left(k_{i}(t)\right)\right) u\left(k_{i}(t)\right)+\right. \\
\left.+\sum_{\substack{j=1 \\
j \neq i}}^{n}\left(\mu_{j}\left(k_{j}(t)\right) p_{j i}+\theta_{j}\left(k_{j}(t)\right) q_{j i}\right) u\left(k_{j}(t)\right)\right] \Delta t+o(\Delta t)
\end{gathered}
$$

on time interval $[t, t+\Delta t)$ there will be no change of system $S_{i}$ nothing is going to happen, $i=\overline{1, n}$.

In addition, for each small time interval $\Delta t$ claim capacity in the system $S_{i}$ increased by a value $r_{i} \Delta t$, where $r_{i}-\mathrm{RV}$ with $E\left\{r_{i}\right\}=c_{i}, i=\overline{1, n}$. We shall also assume, that RV $r_{0 i}, R_{0 i}, r_{i}, r_{j i}, R_{i j}, h_{j i}, H_{i j}$ pairwise independent, $i, j=\overline{1, n}$. Then from the aforesaid follows:

$$
\Delta V_{i}(t, \Delta t)=\left\{\begin{array}{lll}
r_{0 i}+r_{i} \Delta t & \text { with probability } & \lambda p_{0 i} \Delta t+o(\Delta t), \\
-R_{i 0}+r_{i} \Delta t & \text { with probability } & \mu_{i}\left(k_{i}(t)\right) u\left(k_{i}(t)\right) p_{i 0} \Delta t+o(\Delta t), \\
r_{j i}+r_{i} \Delta t & \text { with probability } \mu_{j}\left(k_{j}(t)\right) u\left(k_{j}(t)\right) p_{i j} \Delta t+o(\Delta t), \\
-R_{i j}+r_{i} \Delta t & \text { with probability } \mu_{i}\left(k_{i}(t)\right) u\left(k_{i}(t)\right) p_{i j} \Delta t+o(\Delta t), \\
-H_{i 0}+r_{i} \Delta t & \text { with probability } \theta_{i}\left(k_{i}(t)\right) u\left(k_{i}(t)\right) q_{i 0} \Delta t+o(\Delta t), \\
h_{j i}+r_{i} \Delta t & \text { with probability } \theta_{j}\left(k_{j}(t)\right) u\left(k_{j}(t)\right) q_{j i} \Delta t+o(\Delta t), \\
-H_{i j}+r_{i} \Delta t & \text { with probability } \theta_{i}\left(k_{i}(t)\right) u\left(k_{i}(t)\right) q_{i j} \Delta t+o(\Delta t), \\
r_{i} \Delta t & \text { with probability } \\
& 1-\left[\lambda p_{0 i}+\left(\mu_{i}\left(k_{i}(t)\right)+\theta_{i}\left(k_{i}(t)\right)\right) u\left(k_{i}(t)\right)+\right. \\
& \left.\left.+\sum_{\substack{j=1 \\
j \neq i}}^{n}\left(\mu_{j}\left(k_{j}(t)\right) p_{j i}+\theta_{j}\left(k_{j}(t)\right) q_{j i}\right) u\left(k_{j}(t)\right)\right] \Delta t+o(\Delta t)\right]
\end{array}\right.
$$

At a fixed implementation process $k(t)$ and considering (4) we can write 


$$
\begin{gathered}
E\left\{\Delta V_{i}(t, \Delta t) / k(t)\right\}=\left[\lambda p_{0 i} a_{0 i}-\mu_{i}\left(k_{i}(t)\right) u\left(k_{i}(t)\right)\left(p_{i 0} b_{i 0}+\sum_{\substack{j=1 \\
j \neq i}}^{n} p_{i j} b_{i j}\right)-\right. \\
-\theta_{i}\left(k_{i}(t)\right) u\left(k_{i}(t)\right)\left(q_{i 0} \bar{H}_{i 0}+\sum_{\substack{j=1 \\
j \neq i}}^{n} q_{i j} \bar{H}_{i j}\right)+\sum_{\substack{j=1 \\
j \neq i}}^{n}\left(\mu_{j}\left(k_{j}(t)\right) u\left(k_{j}(t)\right) p_{j i} a_{j i}+\right. \\
\left.\left.+\theta_{j}\left(k_{j}(t)\right) u\left(k_{j}(t)\right) q_{j i} \bar{h}_{j i}\right)\right] \Delta t+o(\Delta t) .
\end{gathered}
$$

Averaging over $k(t)$ and taking into account the normalization condition $\sum_{k} P(k(t)=k)=1$, to change the expected revenue of the system we obtain

$$
\begin{gathered}
E\left\{\Delta V_{i}(t, \Delta t)\right\}=\sum_{k} P(k(t)=k) E\left\{\Delta V_{i}(t, \Delta t) / k(t)\right\}= \\
=\sum_{k_{1}=0}^{\infty} \sum_{2}=0 \\
\infty \\
=\left[\begin{array}{l}
\lambda p_{0 i} a_{0 i}+c_{i}-\left(p_{i 0} b_{i 0}+\sum_{\substack{j=1 \\
j \neq i}}^{n} p_{i j} b_{i j}\right. \\
k_{n}
\end{array}\right) \sum_{k} P\left(k(t)=\left(k_{1}(t), k_{2}(t), \ldots, k_{n}(t)\right)\right) E\left\{\Delta V_{i}(t, \Delta t) / k(t)=\left(k_{1}(t), k_{2}(t), \ldots, k_{n}(t)\right)\right\}= \\
-\left(q_{i 0} \bar{H}_{i 0}+\sum_{\substack{j=1 \\
j \neq i}}^{n} q_{i j} \bar{H}_{i j}\left(k_{i}(t)\right) u\left(k_{i}(t)\right)-\right. \\
+\sum_{\substack{j=1 \\
j \neq i}}^{n} P(k(t)=k) \theta_{i}\left(k_{i}(t)\right) u\left(k_{i}(t)\right)+ \\
\left.\sum_{k} P(k(t)=k)\left(\mu_{j}\left(k_{j}(t)\right) u\left(k_{j}(t)\right) p_{j i} a_{j i}+\theta_{j}\left(k_{j}(t)\right) u\left(k_{j}(t)\right) q_{j i} \bar{h}_{j i}\right)\right] \Delta t+o(\Delta t) .
\end{gathered}
$$

Let the QS $S_{i}$ contains $m_{i}$ identical service lines, in each of which service times of claims and leaving it from the queue distributed exponentially according to the parameters $\mu_{i}$ and $\theta_{i}, i=\overline{1, n}$. In this case we have the expressions

$$
\begin{aligned}
& \mu_{i}\left(k_{i}(t)\right) u\left(k_{i}(t)\right)=\left\{\begin{array}{l}
\mu_{i} k_{i}(t), k_{i}(t) \leq m_{i}, \\
\mu_{i} m_{i}, k_{i}(t)>m_{i},
\end{array}=\mu_{i} \min \left(k_{i}(t), m_{i}\right), i=\overline{1, n},\right. \\
& \theta_{i}\left(k_{i}(t)\right) u\left(k_{i}(t)\right)=\left\{\begin{array}{l}
0, k_{i}(t) \leq m_{i}, \\
\theta_{i}\left(k_{i}(t)-m_{i}\right), k_{i}(t)>m_{i},
\end{array}=\theta_{i}\left(k_{i}(t)-m_{i}\right) u\left(k_{i}(t)-m_{i}\right), i=\overline{1, n},\right.
\end{aligned}
$$


Also we assume that the relations are valid

$$
\begin{aligned}
E \min \left(k_{i}(t), m_{i}\right) & =\min \left(N_{i}(t), m_{i}\right), i=\overline{1, n}, \\
E\left[\left(k_{i}(t)-m_{i}\right) u\left(k_{i}(t)-m_{i}\right)\right] & =\left(N_{i}(t)-m_{i}\right) u\left(N_{i}(t)-m_{i}\right), i=\overline{1, n},
\end{aligned}
$$

where $N_{i}(t)=E\left\{k_{i}(t)\right\}$ - the mean number of claims (waiting and servicing) in the system $S_{i}$ on the time interval $[0, t], i=\overline{1, n}$.

Considering these assumptions, we obtain the following approximate relation

$$
\begin{gathered}
E\left\{\Delta V_{i}(t, \Delta t)\right\}=\left[\lambda p_{0 i} a_{0 i}+c_{i}-\mu_{i}\left(p_{i 0} b_{i 0}+\sum_{\substack{j=1 \\
j \neq i}}^{n} p_{i j} b_{i j}\right) \min \left(N_{i}(t), m_{i}\right)+\right. \\
+\sum_{\substack{j=1 \\
j \neq i}}^{n} \mu_{j} p_{j i} a_{j i} \min \left(N_{j}(t), m_{j}\right)-\theta_{i}\left(q_{i 0} \bar{H}_{i 0}+\sum_{\substack{j=1 \\
j \neq i}}^{n} q_{i j} \bar{H}_{i j}\right)\left(N_{i}(t)-m_{i}\right) u\left(N_{i}(t)-m_{i}\right)+ \\
\left.+\sum_{\substack{j=1 \\
j \neq i}}^{n} \theta_{j} q_{j i} \bar{h}_{j i}\left(N_{j}(t)-m_{j}\right) u\left(N_{j}(t)-m_{j}\right)\right] \Delta t+o(\Delta t) .
\end{gathered}
$$

According to the Poisson process of rate $\lambda$, then the mean number of claims arrived from outside to the system $S_{i}$ during time $\Delta t$ equals $\lambda p_{0 i} \Delta t$. Denote by $\rho(t)$ - the mean number of busy service lines in the system $S_{i}$ at time $t$, $i=\overline{1, n}$. Then $\mu_{i} \rho_{i}(t) \Delta t$ and $\theta_{i}\left(N_{i}(t)-m_{i}\right) u\left(N_{i}(t)-m_{i}\right) \Delta t$ - the mean number of claims, leaving the system $S_{i}$ during time $\Delta t$ respectively, after serving in it and without waiting for the service in it; $\sum_{\substack{j=1 \\ j \neq i}}^{n} \mu_{j} \rho_{j}(t) p_{j i} \Delta t$ and $\sum_{\substack{j=1 \\ j \neq i}}^{n} \theta_{j}\left(N_{j}(t)-m_{j}\right) u\left(N_{j}(t)-m_{j}\right) q_{j i} \Delta t$ the mean number of claims, arriving to the system $S_{i}$ during time $\Delta t$ from other QS respectively, after serving in it and without waiting the service in it. Therefore

$$
\begin{aligned}
N_{i}(t+\Delta t)-N_{i}(t) & =\lambda p_{0 i} \Delta t+\sum_{\substack{j=1 \\
j \neq i}}^{n}\left[\mu_{j} \rho_{j}(t) p_{j i}+\theta_{j}\left(N_{j}(t)-m_{j}\right) u\left(N_{j}(t)-m_{j}\right) q_{j i}\right] \Delta t- \\
- & {\left[\mu_{i} \rho_{i}(t)+\theta_{i}\left(N_{i}(t)-m_{i}\right) u\left(N_{i}(t)-m_{i}\right)\right] \Delta t, i=\overline{1, n}, }
\end{aligned}
$$


where at $\Delta t \rightarrow 0$. It follows the system of ODE for $N_{i}(t)$ :

$$
\begin{gathered}
\frac{d N_{i}(t)}{d t}=\sum_{\substack{j=1 \\
j \neq i}}^{n}\left[\mu_{j} \rho_{j}(t) p_{j i}+\theta_{j}\left(N_{j}(t)-m_{j}\right) u\left(N_{j}(t)-m_{j}\right) q_{j i}\right]- \\
-\mu_{i} \rho_{i}(t)-\theta_{i}\left(N_{i}(t)-m_{i}\right) u\left(N_{i}(t)-m_{i}\right)+\lambda p_{0 i}, i=\overline{1, n} .
\end{gathered}
$$

To find the value $\rho_{i}(t)$ exactly is impossible, therefore, as we have done previously, we approximate its expression

$$
\rho_{i}(t)=\left\{\begin{array}{l}
N_{i}(t), N_{i}(t) \leq m_{i}, \\
m_{i}, N_{i}(t)>m_{i}
\end{array}=\min \left(N_{i}(t), m_{i}\right) .\right.
$$

Then the system of equations (10) takes the form

$$
\begin{gathered}
\frac{d N_{i}(t)}{d t}=\sum_{\substack{j=1 \\
j \neq i}}^{n}\left[\mu_{j} \min \left(N_{j}(t), m_{j}\right) p_{j i}+\theta_{j}\left(N_{j}(t)-m_{j}\right) u\left(N_{j}(t)-m_{j}\right) q_{j i}\right]- \\
-\mu_{i} \min \left(N_{i}(t), m_{i}\right)-\theta_{i}\left(N_{i}(t)-m_{i}\right) u\left(N_{i}(t)-m_{i}\right)+\lambda p_{0 i}, i=\overline{1, n} .
\end{gathered}
$$

That is a system of inhomogeneous linear ODE with discontinuous right-hand sides. It should be solved by dividing the phase space into a number of areas and finding solutions to each of them.

We introduce the notation $v_{i}(t)=M\left\{V_{i}(t)\right\}$. From (1) follows $v_{i}(t+\Delta t)=v_{i}(t)+M\left\{\Delta V_{i}(t, \Delta t)\right\}$. Considering (9), (2), (3), and passing to the limit $\Delta t \rightarrow 0$, we have linear inhomogeneous first order ODE

$$
\begin{gathered}
\frac{d v_{i}(t)}{d t}=-\left[\mu_{i}\left(p_{i 0} b_{i 0}+\sum_{\substack{j=1 \\
j \neq i}}^{n} p_{i j} a_{i j}\right) \min \left(N_{i}(t), m_{i}\right)+\right. \\
+\theta_{i}\left(q_{i 0} \bar{H}_{i 0}+\sum_{\substack{j=1 \\
j \neq i}}^{n} q_{i j} \bar{h}_{j i}\right)\left(N_{i}(t)-m_{i}\right) u\left(N_{i}(t)-m_{i}\right)+ \\
+\sum_{\substack{j=1 \\
j \neq i}}^{n}\left[\mu_{j} p_{j i} a_{j i} \min \left(N_{j}(t), m_{j}\right)+\theta_{j} q_{j i} \bar{h}_{j i}\left(N_{j}(t)-m_{j}\right)\left(N_{j}(t)-m_{j}\right)\right]+\lambda p_{0 i} a_{0 i}+c_{i} .(12)
\end{gathered}
$$

By setting the initial conditions $v_{i}(0)=v_{i 0}, i=\overline{1, n}$, we can find the total expected capacity of claims in network systems. 
If the network operates as there are no observed queues in the average (lowtraffic regime), i.e. $\min \left(N_{i}(t), m_{i}\right)=N_{i}(t), u\left(N_{i}(t)-m_{i}\right)=0, i=\overline{1, n}$, then relations (11) and (12) will have the form:

$$
\begin{gathered}
\frac{d N_{i}(t)}{d t}=\sum_{\substack{j=1 \\
j \neq i}}^{n} \mu_{j} p_{j i} N_{j}(t)-\mu_{i} N_{i}(t)+\lambda p_{0 i}, i=\overline{1, n}, \\
\left\{\begin{array}{l}
\frac{d v_{i}(t)}{d t}=-\mu_{i}\left(p_{i 0} b_{i 0}+\sum_{\substack{j=1 \\
j \neq i}}^{n} p_{i j} b_{i j}\right) N_{i}(t)+\sum_{\substack{j=1 \\
j \neq i}}^{n} \mu_{j} p_{j i} a_{j i} N_{j}(t)+\lambda p_{0 i} a_{0 i}+c_{i}, \\
v_{i}(0)=v_{i 0}, i=\overline{1, n} .
\end{array}\right.
\end{gathered}
$$

If QS operates under a heavy-traffic regime, then $\min \left(N_{i}(t), m_{i}\right)=m_{i}$, $u\left(N_{i}(t)-m_{i}\right)=1, i=\overline{1, n}$ and relations (11), (12) take the form

$$
\begin{gathered}
\frac{d N_{i}(t)}{d t}=-\theta_{i} N_{i}(t)+\sum_{\substack{j=1 \\
j \neq i}}^{n} \theta_{j} q_{j i} N_{j}(t)+ \\
+\sum_{\substack{j=1 \\
j \neq i}}^{n}\left(\mu_{j} p_{j i}-\theta_{j} q_{j i}\right) m_{j}-\left(\mu_{i}-\theta_{i}\right) m_{i}+\lambda p_{0 i}, i=\overline{1, n}, \\
\frac{d v_{i}(t)}{d t}=-\left[\begin{array}{c}
\left.\mu_{i}\left(p_{i 0} b_{i 0}+\sum_{\substack{j=1 \\
j \neq i}}^{n} p_{i j} a_{i j}\right) m_{i}+\theta_{i}\left(q_{i 0} \bar{H}_{i 0}+\sum_{\substack{j=1 \\
j \neq i}}^{n} q_{i j} \bar{h}_{j i}\right)\left(N_{i}(t)-m_{i}\right)\right]+ \\
+\sum_{\substack{j=1 \\
j \neq i}}^{n}\left[\mu_{j} p_{j i} a_{j i} m_{j}+\theta_{j} q_{j i} \bar{h}_{j i}\left(N_{j}(t)-m_{j}\right)\right]+\lambda p_{0 i} a_{0 i}+c_{i}, \\
v_{i}(0)=v_{i 0}, i=\overline{1, n} .
\end{array}\right.
\end{gathered}
$$

The system (15) can be rewritten in matrix form $\frac{d N(t)}{d t}=D N(t)+f$, where: $N^{T}(t)=\left(N_{1}(t), N_{2}(t), \ldots N_{n}(t)\right), \quad D \quad$ - square matrix consisting of elements $d_{i j}=\theta_{j} q_{j i}, \quad i, j=\overline{1, n}, f-$ a column vector whose elements are the values 
$f_{i}=\lambda p_{0 i}+\sum_{j=1}^{n}\left(\mu_{j} p_{j i}-\theta_{j} q_{j i}\right) m_{j}, \quad p_{i i}=q_{i i}=-1, \quad i=\overline{1, n}$. The solution of the last system has the form $N(t)=N(0) e^{D t}+f \int_{0}^{t} e^{D(t-\tau)} d \tau$, where $N(0)$ - given initial conditions.

\section{Expected revenues of the systems in the closed network with central QS}

Consider a closed network with a central QS (Fig. 1). All queueing systems operate under a heavy-traffic regime, i.e. $\forall t>0 \quad k_{i}(t)>0, i=\overline{1, n}$. In this case $\min \left(N_{i}(t), m_{i}\right)=m_{i}, i=\overline{1, n}$. Claims without waiting for service can only move between the peripheral QS and leave the central QS.

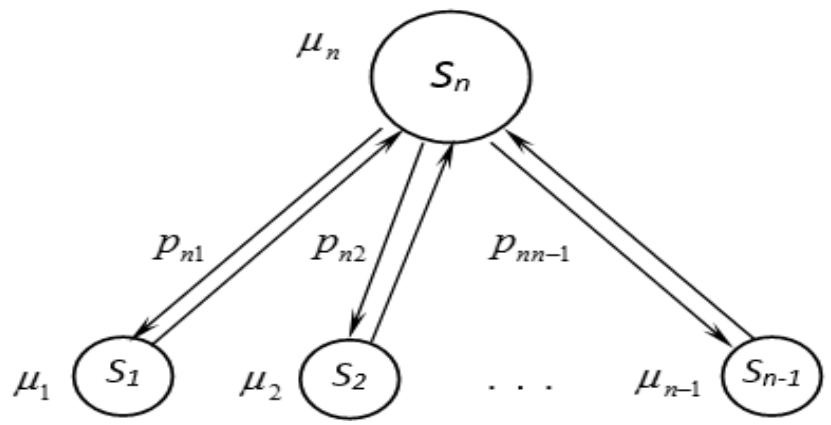

Fig. 1. The closed network with a central QS

Thus $\quad p_{i j}=\left\{\begin{array}{l}p_{n j}, i=n, j=\overline{1, n-1}, \\ 0, i \neq n, j=\overline{1, n-1}, \\ 1, \quad i=\overline{1, n-1}, j=n,\end{array} \quad q_{i j}=\left\{\begin{array}{l}q_{n j}, i=n, j=\overline{1, n-1}, \\ q_{i j}, i, j=\overline{1, n-1}, i \neq j, \\ 0, \quad i=\overline{1, n-1}, j=n .\end{array}\right.\right.$

The system (11) then has the form

$$
\left\{\begin{array}{l}
\frac{d N_{i}(t)}{d t}=-\theta_{i} N_{i}(t)+\sum_{\substack{j=1 \\
j \neq i}}^{n} \theta_{j} q_{j i} N_{j}(t)-\sum_{\substack{j=1 \\
j \neq i}}^{n} \theta_{j} q_{j i} m_{j}-\left(\mu_{i}-\theta_{i}\right) m_{i}+\mu_{n} p_{n i} m_{n}, \\
\frac{d N_{n}(t)}{d t}=-\theta_{n} N_{n}(t)-\left(\mu_{n}-\theta_{n}\right) m_{n}+\sum_{j=1}^{n-1} \mu_{j} m_{j}, i=\overline{1, n-1,}
\end{array}\right.
$$


and $\sum_{i=1}^{n} N_{i}(t)=K$, where $K$ - claims count in the network. The system (16) for the expected revenues can be written as:

$$
\left\{\begin{array}{c}
\frac{d v_{i}(t)}{d t}=-\theta_{i} \sum_{\substack{j=1 \\
j \neq i}}^{n-1} q_{i j} \bar{h}_{j i} N_{i}(t)+\sum_{\substack{j=1 \\
j \neq i}}^{n} \theta_{j} q_{j i} \bar{h}_{j i} N_{j}(t)+\theta_{i} \sum_{\substack{j=1 \\
j \neq i}}^{n-1} q_{i j} \bar{h}_{j i} m_{i}- \\
-\sum_{\substack{j=1 \\
j \neq i}}^{n} \theta_{j} q_{j i} \bar{h}_{j i} m_{j}+\mu_{n} p_{n i} a_{n i} m_{n}-\mu_{i} a_{i n} m_{i}+c_{i}, \quad i=\overline{1, n-1}, \\
\frac{d v_{n}(t)}{d t}=\theta_{n} \sum_{j=1}^{n-1} q_{n j} \bar{h}_{j n} N_{n}(t)+\sum_{j=1}^{n-1}\left(\theta_{n} q_{n j} \bar{h}_{j n} m_{n}+\mu_{j} a_{n j} m_{j}-\mu_{n} p_{n j} a_{j n} m_{n}\right)+c_{n} .
\end{array}\right.
$$

Then, for example, the mean number of claims in the central QS on initial conditions $N_{n}(0)$, equal

$$
N_{n}(t)=-\frac{\mu_{n} m_{n}}{\theta_{n}}+m_{n}+\frac{1}{\theta_{n}} \sum_{j=1}^{n-1} \mu_{j} m_{j}+e^{\theta_{n} t}\left(N_{n}(0)+\left(\mu_{n}-\theta_{n}\right) \frac{m_{n}}{\theta_{n}}-\frac{1}{\theta_{n}} \sum_{j=1}^{n-1} \mu_{j} m_{j}\right),
$$

and expected revenue on initial conditions $v_{n}(0)=v_{n 0}$, takes the form

$$
\begin{gathered}
v_{n}(t)=v_{n 0}+\sum_{j=1}^{n-1} q_{n j} \bar{h}_{j n}\left[\left(\theta_{n} m_{n}+\sum_{j=1}^{n-1} \mu_{j} m_{j}\right) t-\right. \\
\left.+\mathrm{e}^{-\theta_{n} t}\left(N_{n}(0)-\left(\mu_{n}-\theta_{n}\right) \frac{m_{n}}{\theta_{n}}-\frac{1}{\theta_{n}} \sum_{j=1}^{n-1} \mu_{j} m_{j}\right)\right]+ \\
+\left[\sum_{j=1}^{n-1}\left(\theta_{n} m_{n} q_{n j} \bar{h}_{j n}+\mu_{j} a_{n j} m_{j}-\mu_{n} m_{n} p_{n j} a_{j n}\right)+c_{n}\right] t+ \\
+\sum_{j=1}^{n-1} q_{n j} \bar{h}_{j n}\left(N_{n}(0)-\frac{m_{n}}{\theta_{n}}\left(\mu_{n}-\theta_{n}\right)-\frac{1}{\theta_{n}} \sum_{j=1}^{n-1} \mu_{j} m_{j}\right) .
\end{gathered}
$$

\section{Model example}

Consider a closed network with a central QS consisting of $n=6$ QS, $m_{1}=3$, $m_{2}=m_{5}=2, m_{3}=m_{4}=5, m_{6}=20, K=100$. Service rates of claims equal: $\mu_{1}=5$, $\mu_{2}=\mu_{3}=4, \mu_{4}=2, \mu_{5}=3, \mu_{6}=15$. The mean duration of waiting claims in the 
queue $\mathrm{QS}: \theta_{1}^{-1}=0.167, \theta_{2}^{-1}=0.125, \theta_{3}^{-1}=0.222, \theta_{4}^{-1}=0.385, \theta_{5}^{-1}=0.182$, $\theta_{6}^{-1}=0.167$. Transition probabilities of claims between network QS - $p_{6 i}=1 / 5$, $p_{i 6}=1, i=\overline{1,5}$; let also $p_{i i}=-1, i=\overline{1,6}$; other $p_{i j}=0, i, j=\overline{1,6}$. Transition probabilities of claims without waiting for service between network QS: $q_{12}=q_{25}=q_{31}=q_{45}=q_{52}=2 / 3, q_{14}=q_{23}=q_{34}=q_{41}=q_{52}=1 / 3, q_{6 i}=1 / 5, \quad i=\overline{1,5}$, other $q_{i j}=0$, a $q_{i i}=-1 i, j=\overline{1,6}$. Let also $N_{i}(0)=16, i=\overline{1,5}, N_{6}(0)=20$. We define the values for the required expectations:

$$
\begin{gathered}
a_{6 i}=a_{i 6}=(0.3,0.4,1,0.9,1.6), i=1,5, \bar{h}_{6 i}=\bar{h}_{i 6}=(0.3,0.5,0.6,0.8,0.7), i=\overline{1,5}, \\
c=(11,20,20,14,12,17) .
\end{gathered}
$$

Solving the system (17) by the direct method, we get the expression for the mean number of claims in each of the network systems

$$
\begin{gathered}
\begin{array}{r}
N_{1}(t)=10.024-8.224 e^{-6.522 t}+e^{-4.501 t}(4.809 \sin 0.766 t+5.933 \cos 0.766 t)+ \\
+e^{-8.538 t}(0.267 \cos 1.139 t+1.114 \sin 1.139 t),
\end{array} \\
\begin{array}{r}
N_{2}(t)=11.234- \\
-5.151 e^{-6.522 t}+e^{-4.501 t}(1.575 \sin 0.766 t+3.084 \cos 0.766 t)+ \\
+e^{-8.538 t}(3.551 \sin 1.139 t-1.176 \cos 1.139 t),
\end{array} \\
\begin{array}{r}
N_{3}(t)=11.087-9.185 e^{-6.522 t}+e^{-4.501 t}(4.112 \sin 0.766 t+8.746 \cos 0.766 t)- \\
-e^{-8.538 t}(3.768 \sin 1.139 t+2.647 \cos 1.139 t),
\end{array} \\
N_{4}(t)=19.584-0.531 e^{-6.522 t}+e^{-4.501 t}(1.418 \sin 0.766 t-9.897 \cos 0.766 t)- \\
-e^{-8.538 t}(0.677 \sin 1.139 t+1.157 \cos 1.139 t), \\
-e^{-8.538 t}(7.52 \sin 1.139 t+3.233 \cos 1.139 t), \\
N_{6}(t)=29.167-9.167 e^{-6 t} .
\end{gathered}
$$

In solving the system (18) with initial conditions $v_{i}(0)=25, i=\overline{1,5}, v_{6}(0)=50$, we obtained the expressions for the expected claim capacities:

$$
\begin{gathered}
v_{1}(t)=0.55 e^{-6 t}+33.92 t+24.45, \quad v_{2}(t)=0.917 e^{-6 t}+57.1 t+24.083, \\
v_{3}(t)=1.1 e^{-6 t}+82.12 t+23.9, \quad v_{4}(t)=1.467 e^{-6 t}+82.2 t+23.533,
\end{gathered}
$$




$$
\begin{gathered}
v_{5}(t)=1.283 e^{-6 t}+121.22 t+23.717, \\
v_{6}(t)=\left\{\begin{array}{l}
5.317 e^{-6 t}-21.6 t+44.683,21.6 t-5.317 e^{-6 t} \leq 44.683 \\
0,21.6 t-5.317 e^{-6 t}>44.683 .
\end{array} .\right.
\end{gathered}
$$

Charts of change in the expected capacity of claims in the network systems are shown in Figures 2 and 3.

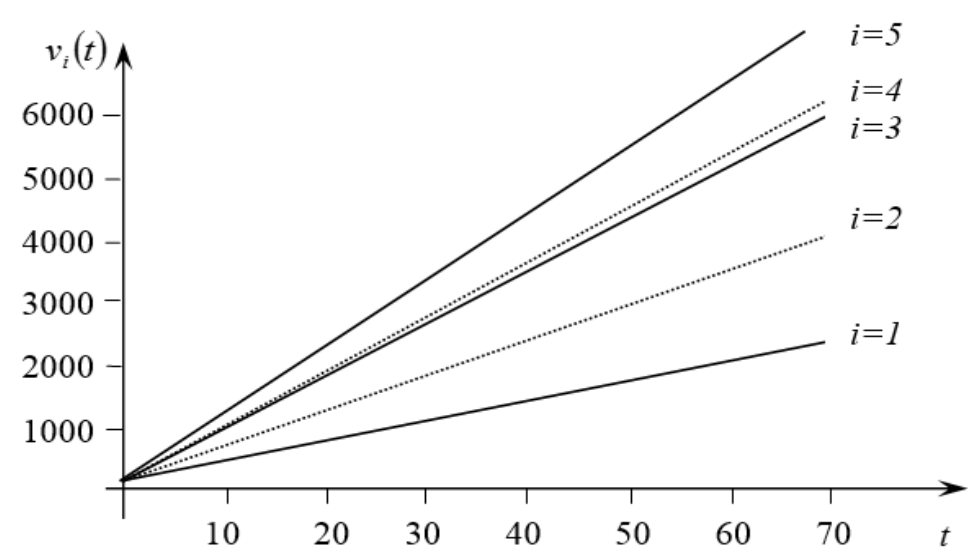

Fig. 2. Expected claims capacity in the systems $S_{i}, i=\overline{1,5}$, of the network

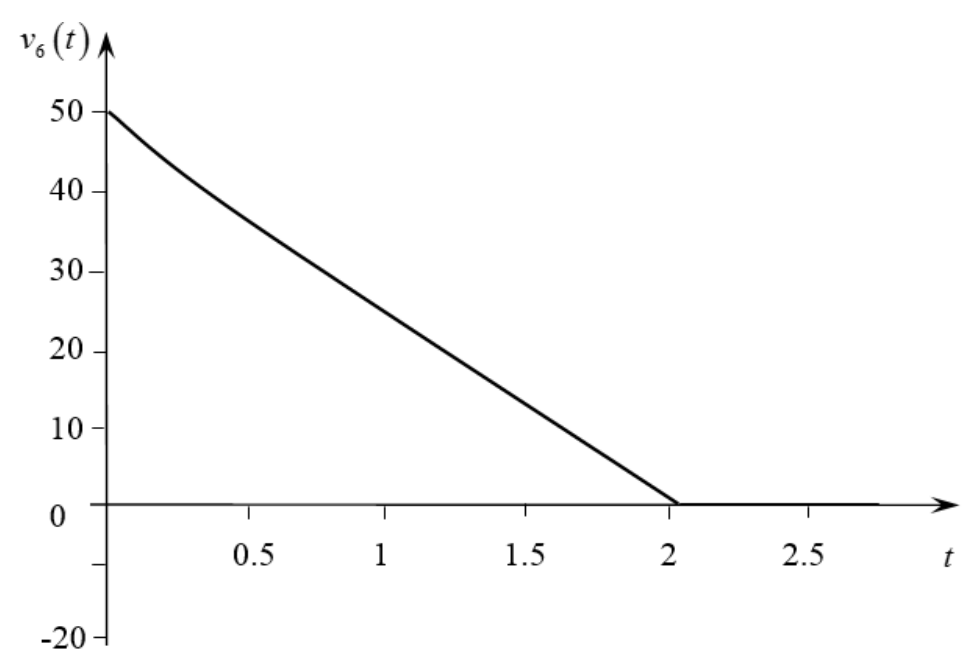

Fig. 3. Expected claims capacity in the central QS $S_{6}$ 


\section{Conclusions}

Further investigations in this area may be associated with the analysis of arbitrary (non-Markov) networks with claims of random capacity and Markov networks with different features, for example, with unreliable service systems, etc.

\section{References}

[1] Tikhonenko O., Metody probalistyczne analizy systemov informacyjnych, Akademicka Oficyna Wydawnicza EXIT, Warszawa 2006.

[2] Matalytski M., Tikhonenko O., Koluzaeva E., Systems and queueing networks: analysis and application [In Russian: Sistemy i seti massovogo obsluzhivaniya: analiz i primeneniya]. Monograph, GrSU, Grodno 2011.

[3] Bufferbloat [Electronic resource]: https://en.wikipedia.org/wiki/Bufferbloat.

[4] Matalytski M., Statkevich S., Stochastic networks with bounded waiting time of claims and unreliable service [In Russian: Stokhasticheskiye seti s ogranichennym vremenem ozhidaniya zayavok i nenadezhnym obsluzhivaniyem], Monograph, GrSU, Grodno 2014.

[5] Matalytski M., Naumenko V., Stochastic networks with non-standard customers movement. [In Russian: Stokhasticheskiye seti s nestandartnymi peremeshcheniyami zayavok]. Monograph, GrSU, Grodno 2016.

[6] Matalytski M., Statkevich S., HM-network analysis with a limited waiting time and random incomes from transitions between states [In Russian: Analiz NM-seti s ogranichennym vremenem ozhidaniya i sluchaynymi dokhodami ot perekhodov mezhdu sostoyaniyami], Vestnik of GrSU, 2009, Ser 2, 3, 6-13.

[7] Matalytski M., Analysis and forecasting of expected incomes in Markov network with bounded waiting time for the claims, Automation and Remote Control 2015, 76(6), 1005-1017.

[8] Matalytski M., Naumenko V., Zastosowanie HM-sieci kolejkowych dla wyznaczenia objetosci pamieci systemow informacyjnych, Studia Informatica 2014, 35(3), 63-69.

[9] Zajac P., Matalytski M., Finding of expected volumes of request in systems of the queueing network with limited amount of spaces of expectation, Studia Informatica 2016, 37(4), 89-104. 